\title{
RADIO GALAXIES AND QUASARS, II
}

Invited Discourse C given on August 28, 1967 in the Lucerna Hall

\author{
Dr. A. SANDAGE \\ (Mount Wilson and Palomar Observatories, Carnegie Institution of Washington, \\ California Institute of Technology, Pasadena, Calif., U.S.A.)
}

\section{The Present Status}

It is my pleasant duty and high privilege to continue the story of radio galaxies and quasars begun by Professor Ryle. Because the intenseness of activity has been so great, and the advance of knowledge so remarkably rapid in this field, it is surprising to recall that only 9 quasars were known at the time of the Hamburg meeting of the Union just 3 years ago. Several hundred are now known, and techniques exist for the discovery of thousands more at will. At the time of Hamburg, less than a year had passed since M. Schmidt's discovery of the red-shift in 3C 273. Hoffleit and Smith, and Matthews and myself had only just established the optical variation of $3 \mathrm{C} 273$ and $3 \mathrm{C} 48$, respectively. There was no knowledge of how extensive the phenomenon of optical variability was, nor of the exceedingly short time-scales of the variation. Ideas of the number of quasars, radio and non-radio, were not yet formed, and the feature of radio doubling had not yet been established as a general characteristic.

Now, 3 years later, the whole of the systematic identification of the $3 \mathrm{C} \mathrm{R}$ catalogue has nearly been achieved, red-shifts exist for over 100 quasars, optical variation has been found to be a general phenomenon, the existence of a class of weakly emitting or radio-quiet quasars established, and patterns of similarity between QSS and radio galaxies have emerged.

The initial thrust of this advance was made possible by the final solution to the optical identification problem - a problem which was so discouraging in the 1950's because of the major failure to continue the initial success in identification of such objects as Tau A, Cen A, Cyg A, and Virgo A, each of which had early been associated with very peculiar optical objects. However, once radio-position accuracies became known to $\pm 15^{\prime \prime}$ in both coordinates, as are now routinely available, optical identification proved to be rapid and relatively easy. This fundamental advance, bordering on the spectacular, was due to ingenious new techniques invented by the radio astronomers.

As the identification work proceeded, primarily by the Cambridge group in England, by Mills and Bolton in Australia, by the workers in Nancy, and by such men as Mat-

Perek (ed.), Highlights of Astronomy, 45-70. (C) I.A.U. 
thews and Minkowski in California, the pattern clearly emerged that there were only two types of optical objects associated with radio sources in high galactic latitudes. Galaxies accounted for about $70 \%$ of the identifications, and the starlike quasars made up the remaining $30 \%$.

Most of the galaxies are ellipticals, normal in appearance, often in clusters, and then, invariably among the several brightest cluster members. The quasars are isolated, not obviously in clusters, and have an image diameter below the optical resolution of present techniques $\left(\sim \frac{1}{2}{ }^{\prime \prime}\right)$.

Professor Ryle has emphasized that the available radio data show rather clearly that explosions have taken place in radio sources, be they galaxies or quasars, sending clouds of plasma away from the central body. Although the evidence is not as abundant in optical astronomy, explosive events have, in fact, been directly observed in a few of the nearby optical objects. That the phenomenon of explosion is involved at all is positively proved by the Crab Nebula (3C 144), which was observed to explode by the Chinese and perhaps by the Arizona American Indians (Miller, 1955) in 1054 A. D.

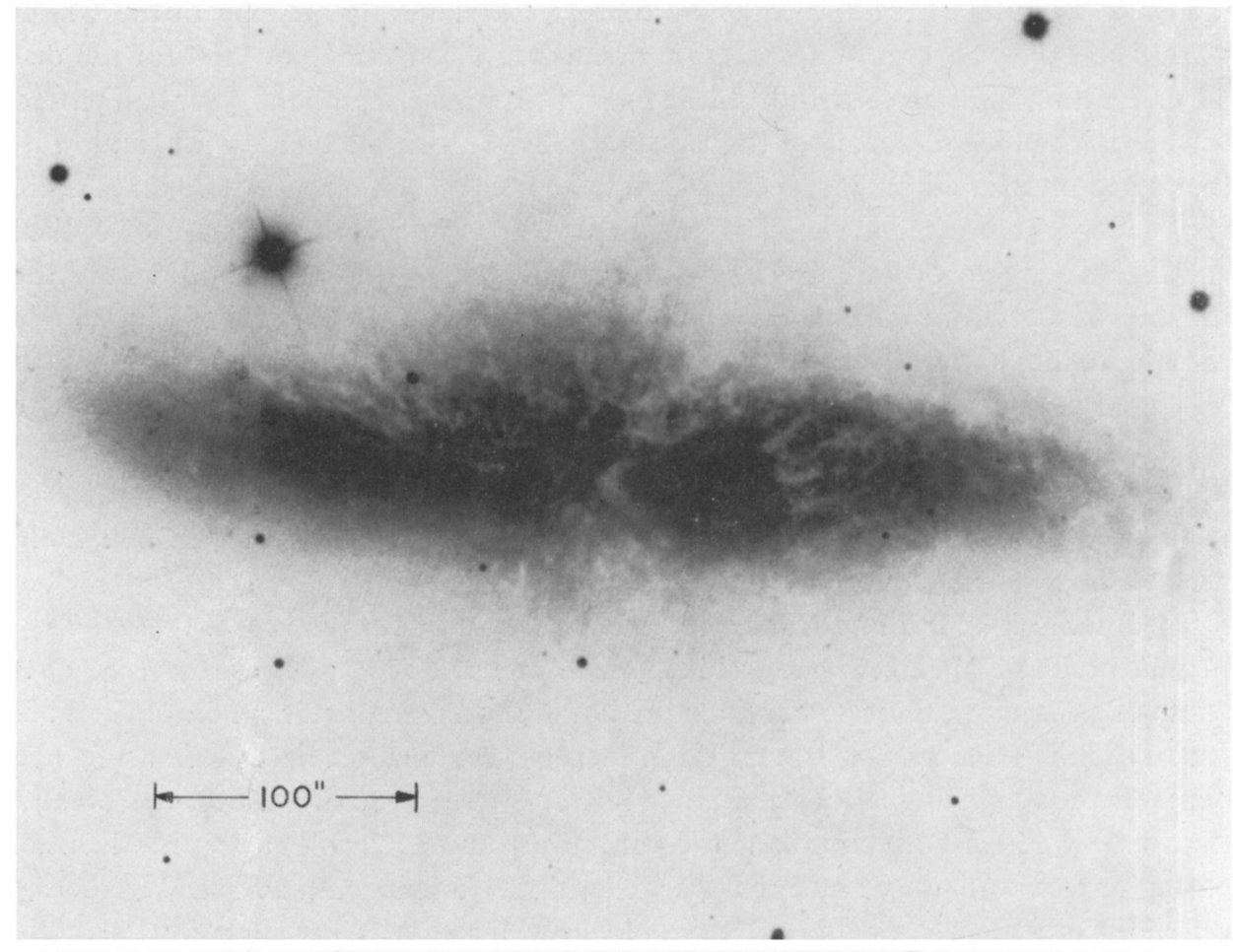

FIG. 1. Photograph in blue light of the exploding galaxy M82 (3C 23I). A chaotic pattern of dust exists across the face, and suggestions of thin flamentary structures near the direction of the minor axis are present. Picture from a plate of 30-min exposure made with the 200-inch Hale reflector on Eastman $103 a \mathrm{O}$ emulsion. 
Although the Crab is in our own Galaxy, the physics involved in its radio emission undoubtedly has relevance to the larger-scale problem of exploding galaxies themselves.

The most direct evidence for galactic explosions is provided by M 82 (Lynds and Sandage, 1963; Sandage and Miller, 1964) and by NGC 1275 (Burbidge and Burbidge, 1965), where filamentary structures in motion have been observed by a combination of direct photography and spectroscopy. Figures 1,2 , and 3 show the change of character of the optical image of M 82 between a short-exposure blue plate $(\lambda \lambda 3900$ $5000 \AA)$, an $\mathrm{H} \alpha$ plate $(\lambda 6563 \pm 40 \AA)$, and a composite print made by superposition of three blue photographs showing the extended outer filaments. The structures of Figure 3 are very highly polarized and are believed to be caused by optical synchrotron emission. Spectroscopic studies show that the $\mathrm{H} \alpha$ structures are expanding with a linear law and that all the gaseous matter was probably ejected from the central regions of M 82 about 2 million years ago (Lynds and Sandage, 1963; Burbidge et al., 1964).

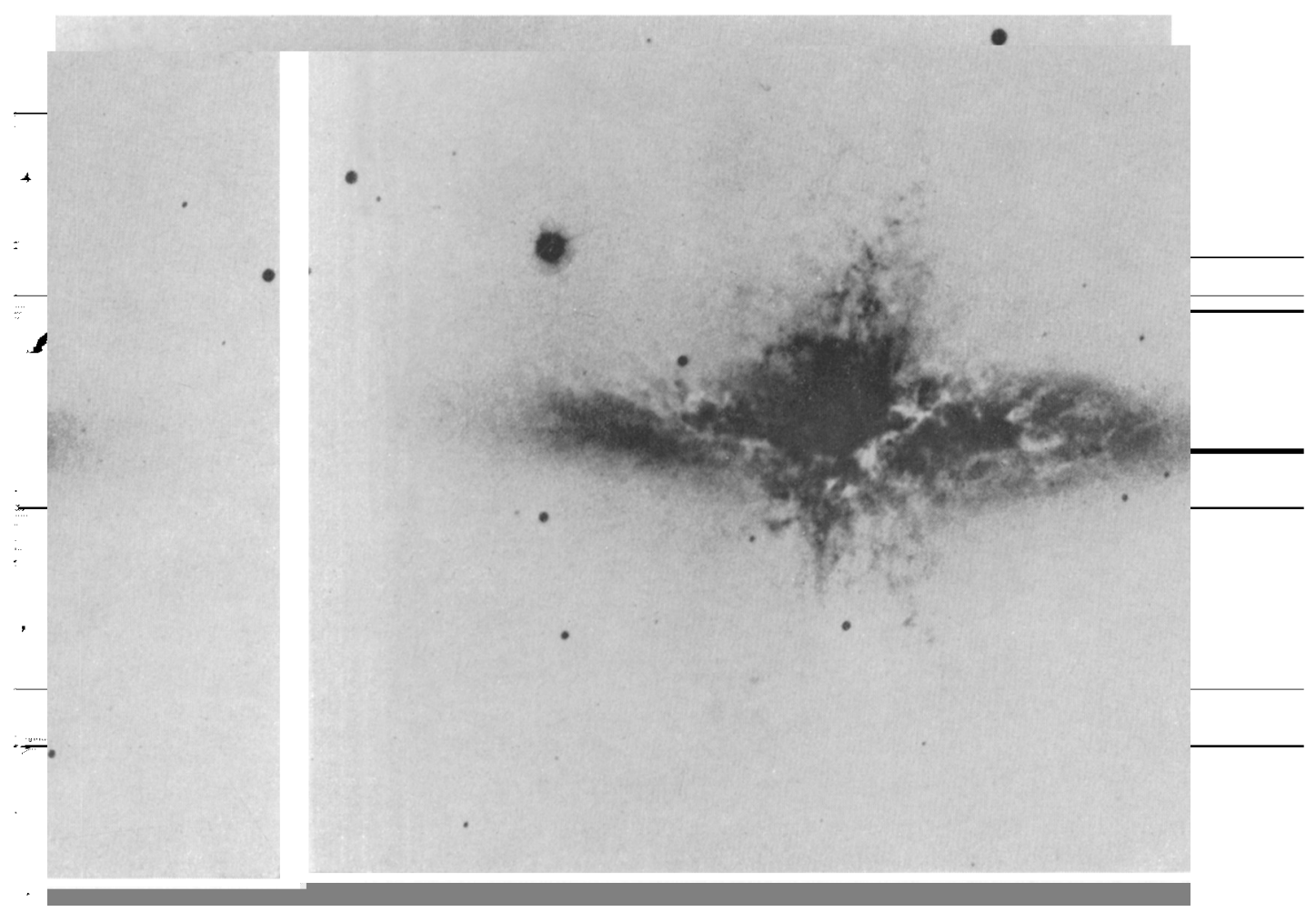




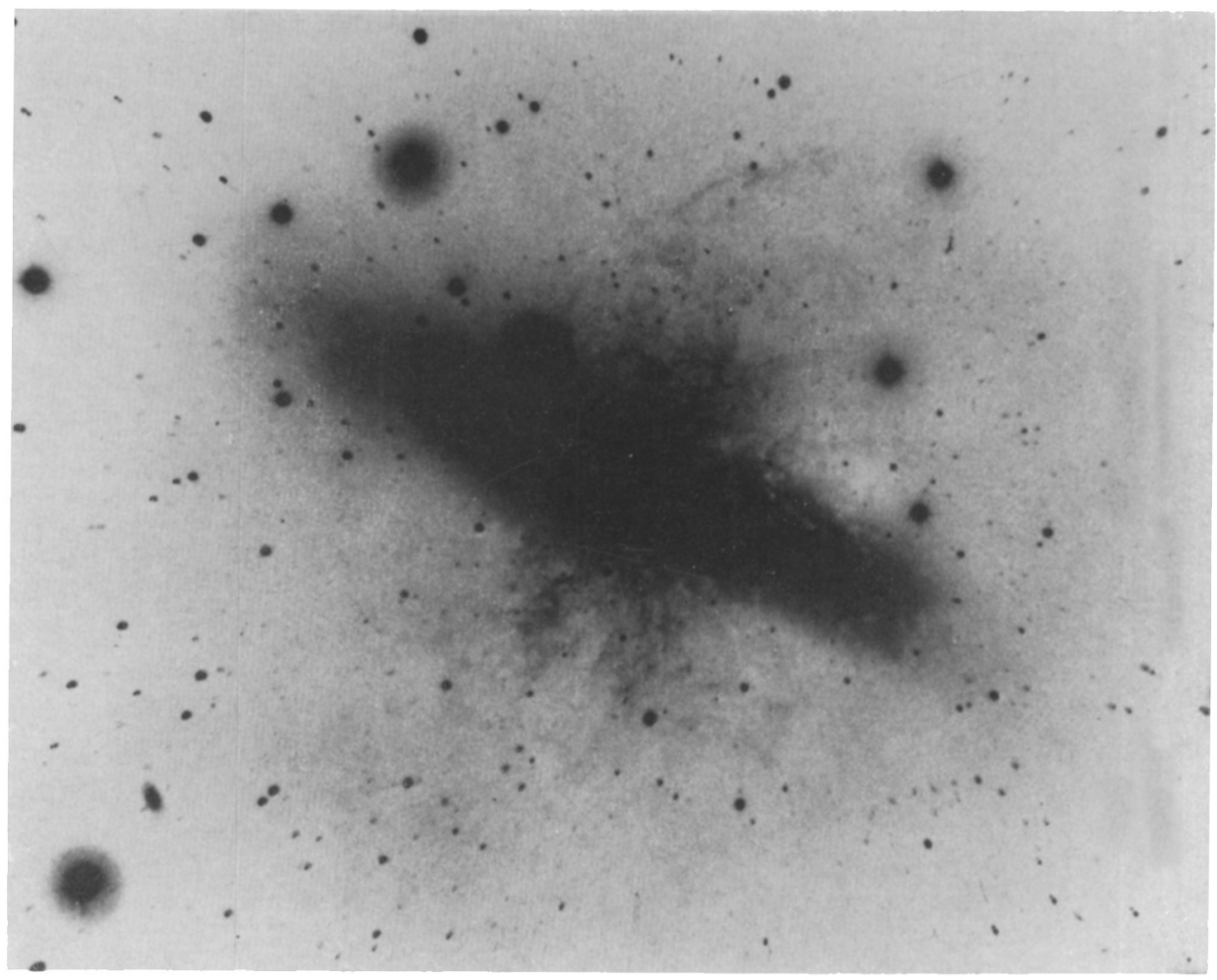

FIG. 3. Composite print of $M 82$ made from three $103 a O$ plates. An extensive set of outer blue filaments is present which emit continuous radiation. The light from these filaments is highly polarized and is believed to be due to optical synchrotron emission (Sandage and Miller, 1964).

The optical forms of most radio galaxies are not as spectacularly peculiar as those for M 82, M 87, NGC 5128, and NGC 1275. The majority of the radio galaxies with radio powers greater than $10^{40} \mathrm{ergs} / \mathrm{sec}$ (in a bandpass from $10^{7}$ to $10^{11} \mathrm{~Hz}$ ) are normal elliptical systems. This fact, established rather late in the identification program, was not fully foreseen because of the obvious peculiarities of the objects initially identified in the 1950's.

The quasars exhibit an entirely different optical appearance from radio galaxies. Figure 4 is a 200 -inch photograph of $3 \mathrm{C} 273$ showing the diffraction pattern of the central image and the well-known optical jet.

Although known since 1960, QSS are still a mystery. Schmidt's identification of the Lyman- $\alpha$ hydrogen line in $3 \mathrm{C} 9$ at an observed wavelength of $\lambda=3666 \AA$, giving $\Delta \lambda / \lambda_{0}=2 \cdot 012$, suggested that quasars partake of the general expansion of the universe. No other explanation for such large apparent velocities had heretofore been successful in a scientific sense, and the evidence is clear and abundant that the red-shifts for normal galaxies are strictly correlated with distance. 
Quasars immediately captured the imagination because, if red-shifts are cosmological, we look back in time some $80 \%$ of the way to the creation event in a Friedmanntype universe for redshifts of $\Delta \lambda / \lambda_{0}=2$. A very early sample of matter is then available, from which deductions can possibly be made of the evolution of the world.

However, if we put the QSS at the Hubble distance, they become the most powerful radiators known. The brightest would emit $10^{47} \mathrm{ergs} / \mathrm{sec}$ over all wavelengths from the radio to the optical, or more than $10^{60} \mathrm{ergs}$ in a lifetime of $10^{6}$ years. And the energy input must be very much more because the conversion processes from injection to radiation are not $100 \%$ efficient. If $10^{62}$ ergs are involved, assuming $1 \%$ efficiency, then $10^{10}$ solar masses must be converted from hydrogen to helium for the most intense QSS.

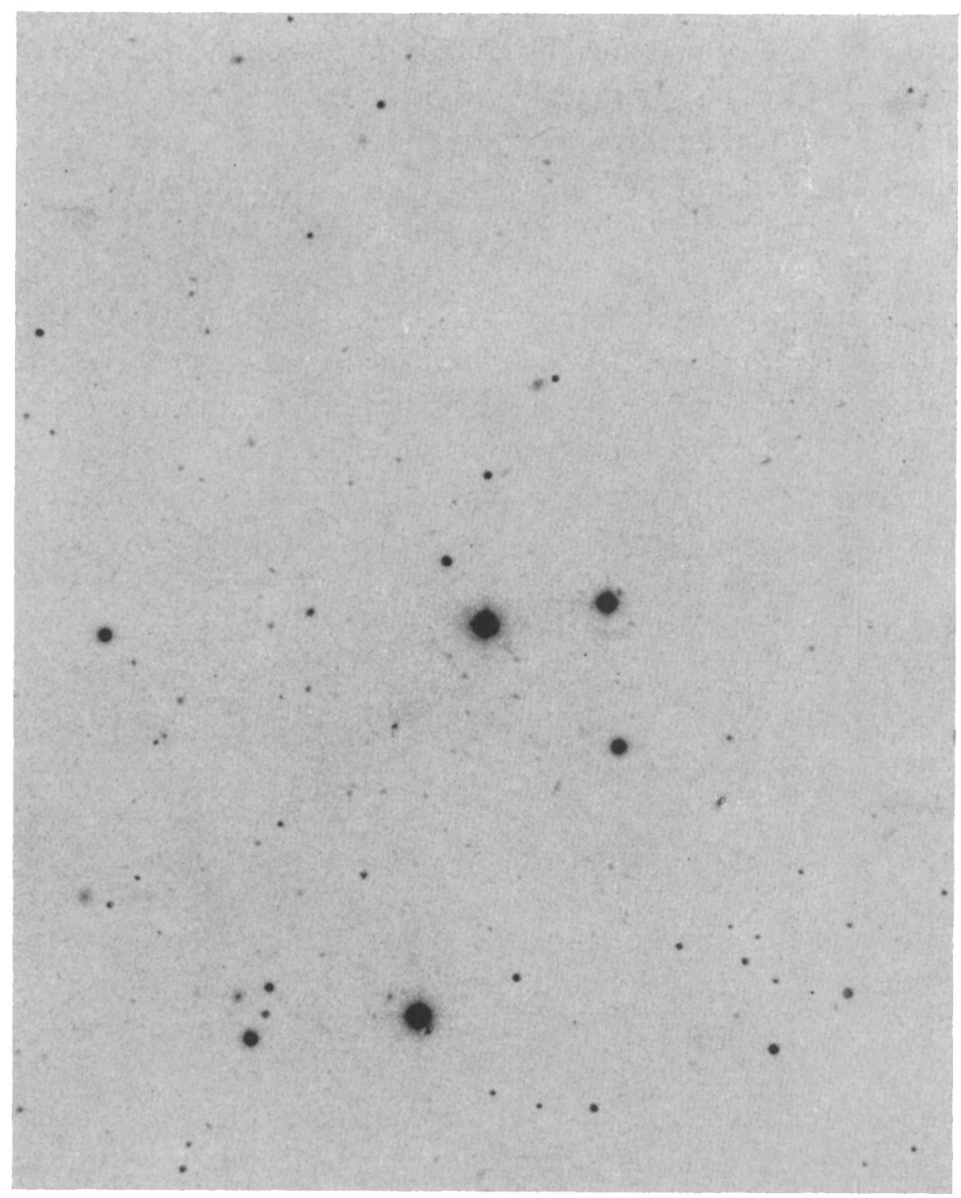

FIG. 4. Photograph of the field of $3 C 273$ from a plate taken with the Hale reflector on Eastman $103 a D$ emulsion of 50-min exposure time, in February 1962. The jet and the diffraction spikes on the central optical image are clearly visible. 
This problem, combined with the consequences of the rapid light variations, has caused some astronomers to doubt the cosmological interpretation of the red-shifts. There is no general agreement. The current debate is like that of the 1920's concerning galaxies as island universes. The subject is the same - that of distance; only the objects are different. In its original version, the debate centered around the distances to galaxies. Today, it is the QSS. Are they local or at the Hubble distance? What is needed now, as then, is a crucial experiment. As echoed in every issue of the current journals, it is clear that either no crucial experiment has yet been performed or, if it has, it has not been so recognized; or, if recognized by some, it is not acknowledged by all.

All versions of all theories can, of course, not be right. Although the evidence seems quite sufficient to eliminate some of the current suggestions, there is still no overwhelming proof of the type presented by Hubble in 1924 on the nature of the galaxies.

However, the evidence just presented by Ryle on the continuity of radio properties between galaxies and quasars, together with other evidence reviewed in later sections here, seem in my opinion, to establish the cosmological interpretation of the redshifts. Conversely, the difficulties in the other interpretations seem, at the moment, paramount.

Questions concerning quasars are of two types. By far the easiest to answer are those of traditional astronomy. These may be called, perhaps unfairly, the superficial problems concerned with such items as the surface distribution over the plane of the sky, the number of QSS, the prevalence and nature of the optical and radio variations, and, most important, their distance. The second class concerns the physics of the situation. What is the energy source? What triggered the explosion? By what mechanism are the electrons accelerated? Can we understand the ontogenetic nature of the event? It is not likely that complete answers to these questions will be found until the astronomical problems are solved. We address ourselves in the following sections to certain questions of the first set, however bringing in problems of physics where answers are available and germane.

\section{Identification and Surface Distribution of QSS}

Extensive optical identification programs have fairly well exhausted the $3 \mathrm{C} \mathrm{R}$ catalogue to the limit of the Palomar 48-inch Schmidt telescope. This work is due to many people at many places, in many countries, and the cordial and enthusiastic international cooperation of radio and optical astronomers clearly marks the result. Following the early classical work of Bolton and Stanley, Mills, Baade and Minkowski, and F.C. Smith, the most important modern names connected with the work are Matthews, Véron, Fomalont, Wyndham, Longair, Parker, Wills, and Dewhirst.

There are 328 radio sources in the $3 \mathrm{C}$ R. The number of confirmed QSS with redshifts and/or UBV photoelectric photometry is 44 . There are a few additional possi- 
bilities which have not yet been completely verified. Of the 328 sources, 229 are in latitudes greater than $\pm 15^{\circ}$. Of these, 41 are confirmed QSS, 38 are empty fields to the limit of the Palomar Schmidt, and 150 are either positive or possible radio galaxies. $27 \%$ of all possible identifications now made in the $3 \mathrm{C} \mathrm{R}$ are QSS. The total number of QSS positively identified, either by spectra or by photometry, in the $3 \mathrm{C}$, the $4 \mathrm{C}$, the Mills, Slee, Hill, and the Parkes catalogs is at least 150 as of May 1967, and the number can apparently be increased at will, especially among the radio-quiet quasars. To July 20, 1967, red-shifts of 103 quasars had been determined, primarily by four extremely active groups of observers which include Schmidt at Mount Wilson and Palomar, Lynds at Kitt Peak, E. M. Burbidge and Kinman at Lick, and Ford and Rubin of the Carnegie Institution.

Figure 5 shows an equal-area projection in galactic coordinates of all known quasars which had been verified to April 1967. There are 134 objects plotted. Dots are QSS from the 3C R, triangles from the Parkes, squares from the $4 \mathrm{C}$ that have been identified by Scheuer and Wills (1966) as blue stellar objects from the Haro-Luyten catalogue, and crosses are radio-quiet quasars found in a special study by Luyten and myself (1967) in the PHL field at $1^{\mathrm{h}} 36^{\mathrm{m}},+6^{\circ}$. The only unbiased sample are the black dots, because identification programs are not yet statistically complete for the Parkes and the $4 \mathrm{C}$ catalogs.

The distribution appears to be isotropic in the area searched north of $\delta=0^{\circ}$ to

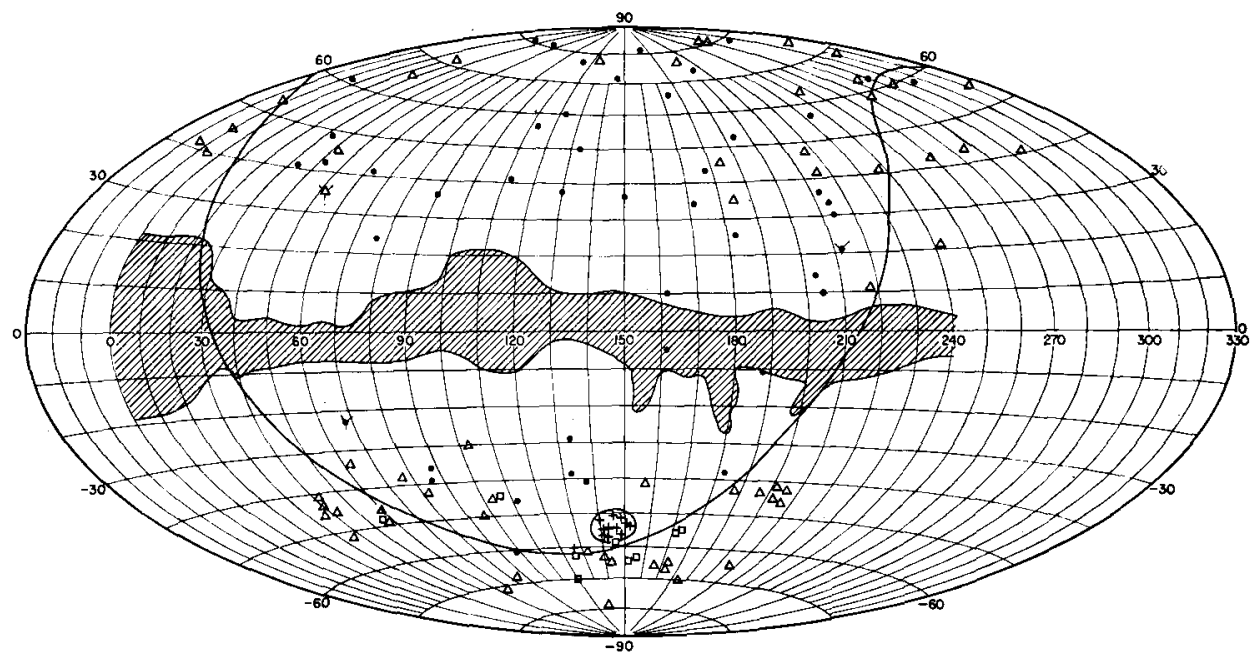

FIG. 5. The distribution in galactic coordinates of quasars known to May 1967. The zone of avoidance in the galactic plane is shown, together with the great circle of zero declination, North of which the $3 C R$ radio catalogue is complete to 9 fux units. Dots represent radio quasars identified from the $3 C R$, triangles are from the Parkes catalogue, squares from the $4 C$ for objects which overlap the PHL catalogue, and crosses are radio-quiet quasars found in a $40 \square^{\circ}$ search area at $1^{\text {h }} 36^{m},+6^{\circ}$. Spikes represent quasars which violate the suggested anisotropy of Strittmatter et al. (1966). 
within the accuracy attainable with the small number of points. There is a general avoidance of the galactic plane, and a relative concentration toward the poles with a roughly cosecant distribution. The crucial conclusion on isotropy can be improved by noting that the Cambridge observers have analyzed segments of the $4 \mathrm{C}$ catalog and found no evidence of anisotropy to the accuracy of their statistics, which is better than $1 \%$. If $30 \%$ of the $4 \mathrm{C}$ class are QSS, then isotropy is assured to better than $3 \%$. This high degree is important for the question of local versus cosmological distances for certain versions of the local hypothesis.

\section{Selected Physical Properties of QSS}

The problem of optical identification of quasars divides into two parts. Those QSS which are strong radio emitters can be, and have been, found by inspection of photographs at the already known radio position on the plane of the sky. However, it now appears that most of the quasars are weak radio emitters and must be found by optical methods, for which knowledge of the optical properties is important. These properties include the nature of the line spectra, the peculiar energy distribution of the optical continuum, and the prevalence of optical variation.

\section{A. OPTICAL LINE SPECTRA}

Most quasars show broad emission lines of both forbidden and permitted transitions. Many lines of the abundant elements are present, such as the Balmer and Lyman series of hydrogen, lines of helium, and the ground-state transitions of carbon, nitrogen, silicon, and magnesium. Forbidden lines of oxygen and neon are often strong.

All emission lines show the same red-shift to within narrow limits. This appears to be a strong argument against the interpretation of the red-shift as due to an intense gravitational field of a single, compact, massive object. The forbidden lines must be formed in regions of low pressure so as to prevent collisional de-excitation of the metastable levels. Such regions, for a single compact body, will almost certainly have a lower gravitational potential than the region of permitted line formation, and hence a smaller red-shift, which is contrary to the well-established fact.

To circumvent this difficulty, Fowler and Hoyle proposed an ad hoc gravitational model where the emission lines originate inside a strong gravitational shell, formed by a uniform clustering of, say, neutron stars. It is here necessary that the gravitational potential-well be exceedingly uniform over the region of line formation to explain the data on the constancy of red-shift from line to line. Furthermore, there is no explanation on this model of the range of redshifts among the quasars as a class, spreading from $z=0.158$ for $3 \mathrm{C} 273$ to larger than 2 for objects such as 3C 9. It is now known (Schmidt, 1968) that the number of quasars at a given redshift, $z$, in a given red-shift range, $\Delta z$, is just that required to explain the $\log N, \log S$ curve for radio galaxies on 
the cosmological hypothesis. This crucial fact must be taken as coincidence on the gravitational-shell model, but, in fact, finds ready explanation on the cosmological hypothesis because radio galaxies are known to be at the Hubble distance (Section 6). This, and the arguments of Greenstein and Schmidt (1964) seem persuasive against the gravitational interpretation.

The emission lines of quasars are usually very broad - often with a $50 \AA$ half-width. Interpreted as a Doppler motion, internal velocities of the order of several thousand $\mathrm{km} / \mathrm{sec}$ are indicated. To stabilize such internal motions in a body of say $0.01 \mathrm{pc}$ radius requires a mass of at least $10^{7} M_{\odot}$, as shown by Setti and Woltjer (1966).

QSS may, of course, not be stable. Evidence comes from the several sources that have absorption lines. Of the 103 QSS with known redshifts, at least 20 have absorption lines, and in many of these cases the absorptions are displaced blueward of the corresponding emission lines, as in P Cyg stars. The simplest explanation is that of an expanding cool envelope with apparent subsequent mass loss. Setti and Woltjer have concluded from the available observations that a minimum initial mass on any assumption of distance is $10^{5} M_{\odot}$ per average QSS - a number which is important in the later discussion of the local vs. the cosmological interpretation of the red-shift.

The most remarkable absorption-line object is PHL 5200 (4C: 05.93) which Lynds (1967) found to have wide, deep absorption bands starting at the emission edges of the lines of Civ (1550), SiIv (1403), and Nv (1243) and extending blueward. These bands are from 100 to $150 \AA$ wide, indicating a continuum of expansion velocities reaching values of about $10000 \mathrm{~km} / \mathrm{sec}$. All other sources studied so far are considerably less extreme.

\section{B. OPTICAL CONTINUUM, COLORS, ENERGY DISTRIBUTION}

The principal distinguishing feature of the observed radiation of QSS is the abnormal ultraviolet flux relative to ordinary stars. This ultraviolet excess was used as a discovery technique from 1964 to 1965 before precise radio positions were available.

Figure 6 shows a conventional two-color diagram for about 100 QSS with colors known to January 1967. $N$-type galaxies, as defined by Matthews et al. (1966), are shown as triangles. Fourteen radio-quiet quasars (QSO) are shown as crosses.

Half of the plotted QSS fall above the black-body line and half below in the region of white dwarfs, U Gem stars, and old novae. Although the distribution looks scattered and random, such is not the case. The red-shifts in various ranges are preferentially concentrated in discrete regions of the diagram, as shown in Figure $7.82 \%$ of the plotted QSS obey this red-shift-area relation. The phenomenon was originally discovered by Kardeshev and Kolmberg from only 16 objects known in March 1966. McCrea and Barnes independently found the same thing in a different representation. Many authors, including Lynds, Schmidt, Strittmatter and G. R. Burbidge, Grewing, Lari and Setti, and myself, have shown that Figure 3 results from features of the energy distribution being shifted into and out of the broad-band U, B, V filters as the red- 


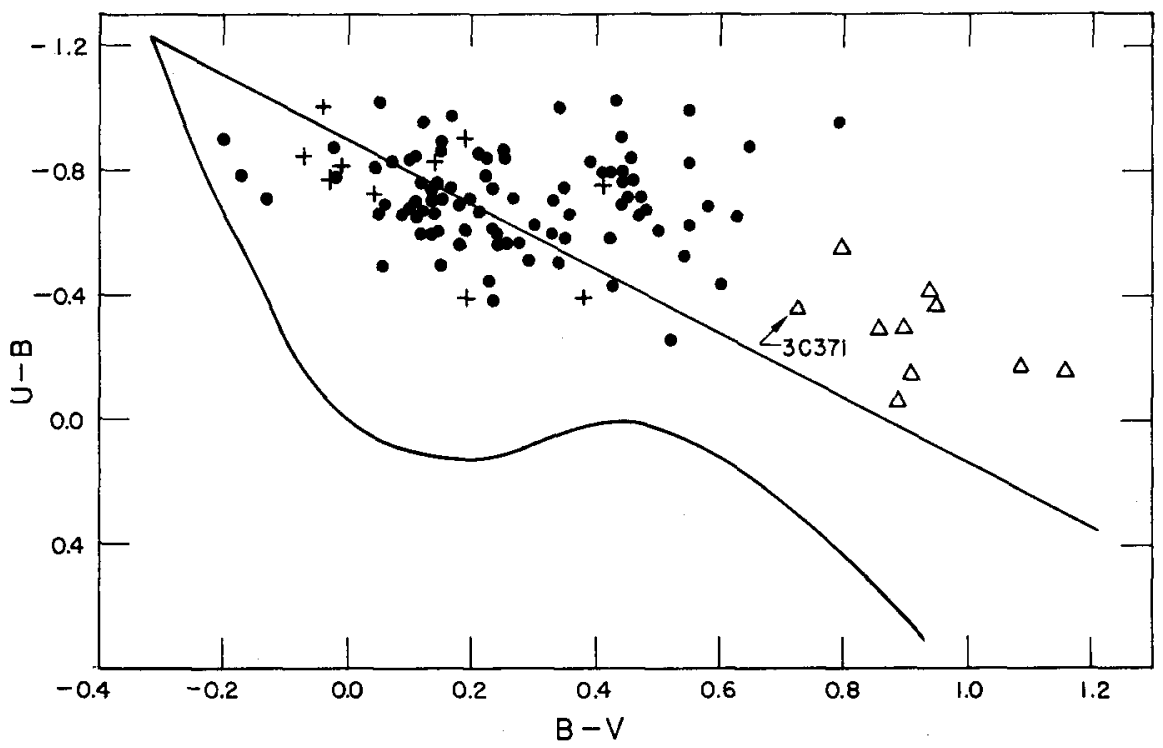

FIG. 6. The two-color diagram for radio quasars (dots), radio-quiet quasars (crosses), and $N$-type galaxies (triangles). The black-body line and the normal stellar relations are shown for comparison.

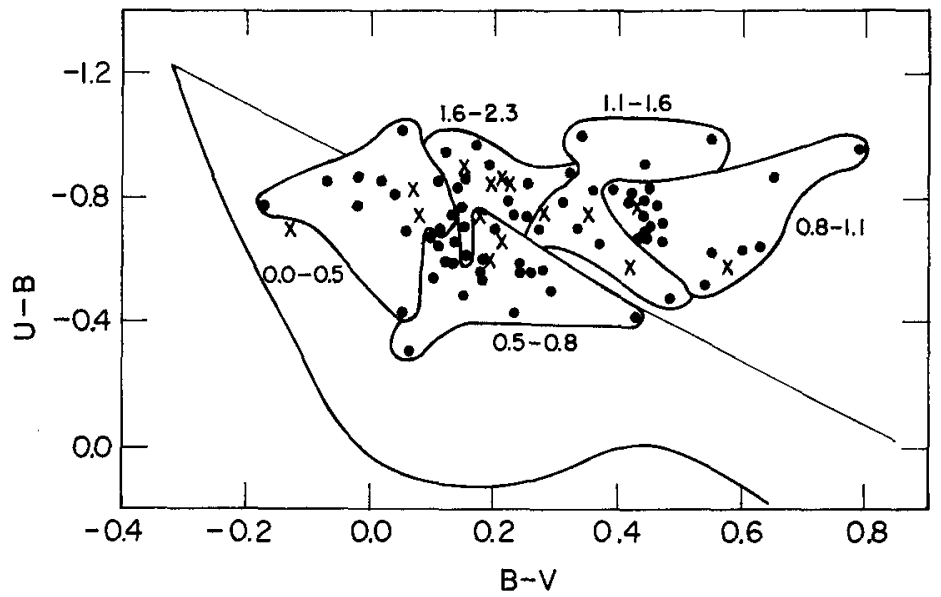

FIG. 7. The two-color diagram for radio quasars of known red-shift. Dots are objects which obey the red-shift-area assignment; crosses are quasars which violate the area designation.

shifts increase. These features are probably lines superposed on a continuum energy distribution which is statistically similar from quasar to quasar.

It is possible to construct a composite $I(\lambda)$ curve for an 'average' QSS from these results (Astrophys. $J ., 146,13,1966$ ). Figure 8 shows a first attempt for the interval $\lambda_{0}=1000 \AA$ to $\lambda_{0}=5000 \AA$. Schmidt, Oke, and Wampler have each also contributed to this problem. 


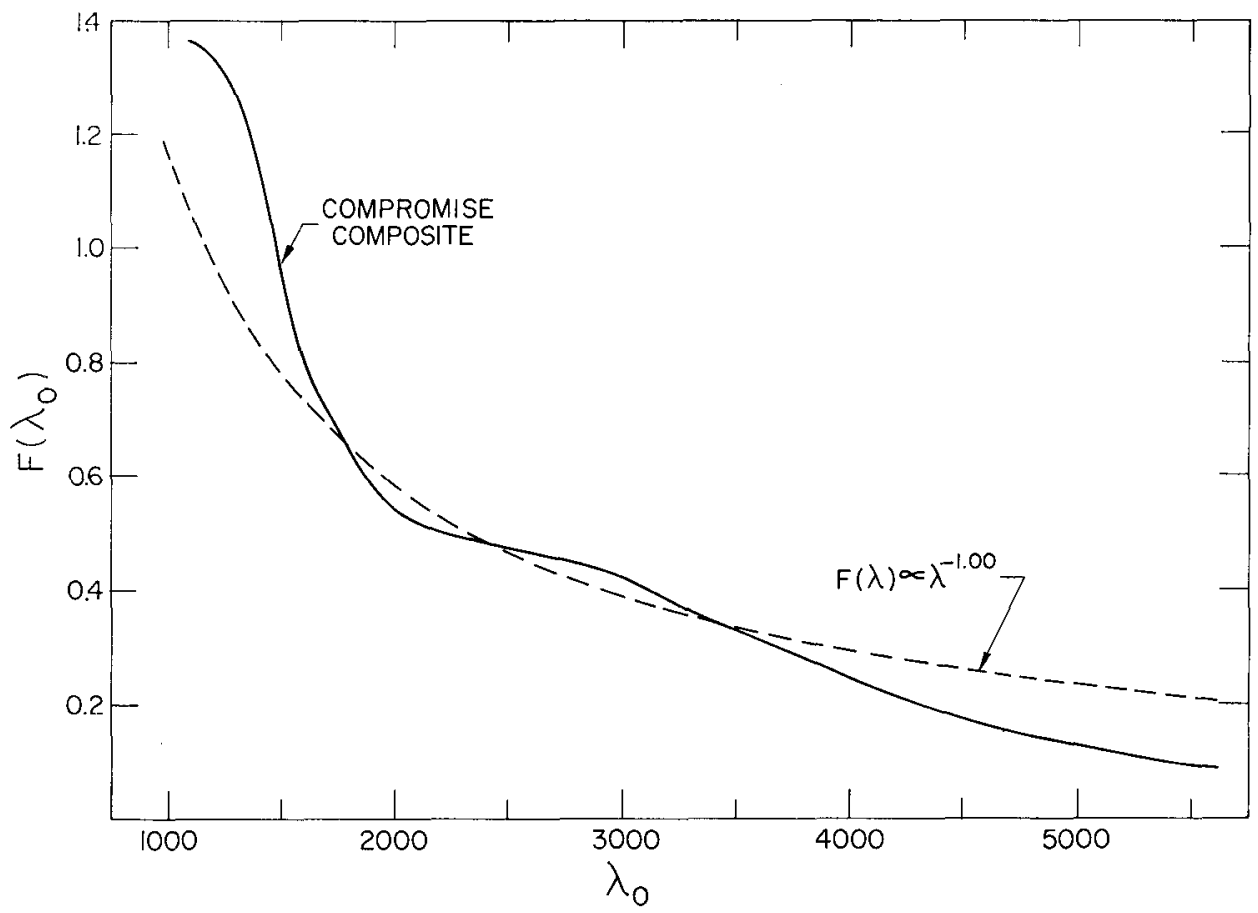

FIG. 8. The energy distribution per unit wavelength in the rest frame for an average quasar in the optical and near $X$-ray region. The dashed curve is a power spectrum, $F(v) \propto v^{-1}$, shown for comparison. The compromise composite represents the true continuum plus line radiation, smoothed by 1000-A bandpass filters characteristic of the UBV system.

The origin of the continuum radiation is still not known with certainty. In the beginning (1963) it seemed possible that the optical flux could be due to the synchrotron process and would connect up simply with the radio spectrum. The best early case was 3C 48, where it was shown (Astrophys. J., 138, 30, 1963) that the measured optical flux from $v=3 \times 10^{14} \mathrm{~Hz}$ to $8 \cdot 3 \times 10^{14} \mathrm{~Hz}(\lambda=10000 \AA$ to $\lambda=3600 \AA)$ agreed with a theoretical synchrotron spectrum (with parameters fitted to the radio data) to better than $\pm 0 \cdot 1$ magnitude, as illustrated in Figures 9 and 10 .

We know now that the situation is much more complex. The results of Low and Johnson (1965) and of Epstein (1965) for 3C 273 show that a simple extrapolation is

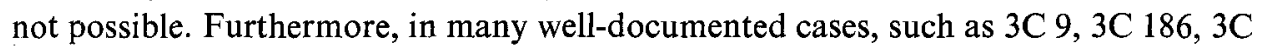
$191,3 \mathrm{C} 204$ and 3C 208, the optical points in the $\log F(v), \log v$ diagram lie as much as a factor of 100 brighter than the expected synchrotron spectrum, as extended by theory from radio frequencies using a monotonic electron-energy distribution. If we are to maintain that the optical radiation is synchrotron for these cases, then at least a tworegime electron-energy distribution is needed.

The crucial observation showing that the radio and optical radiation are not simply 


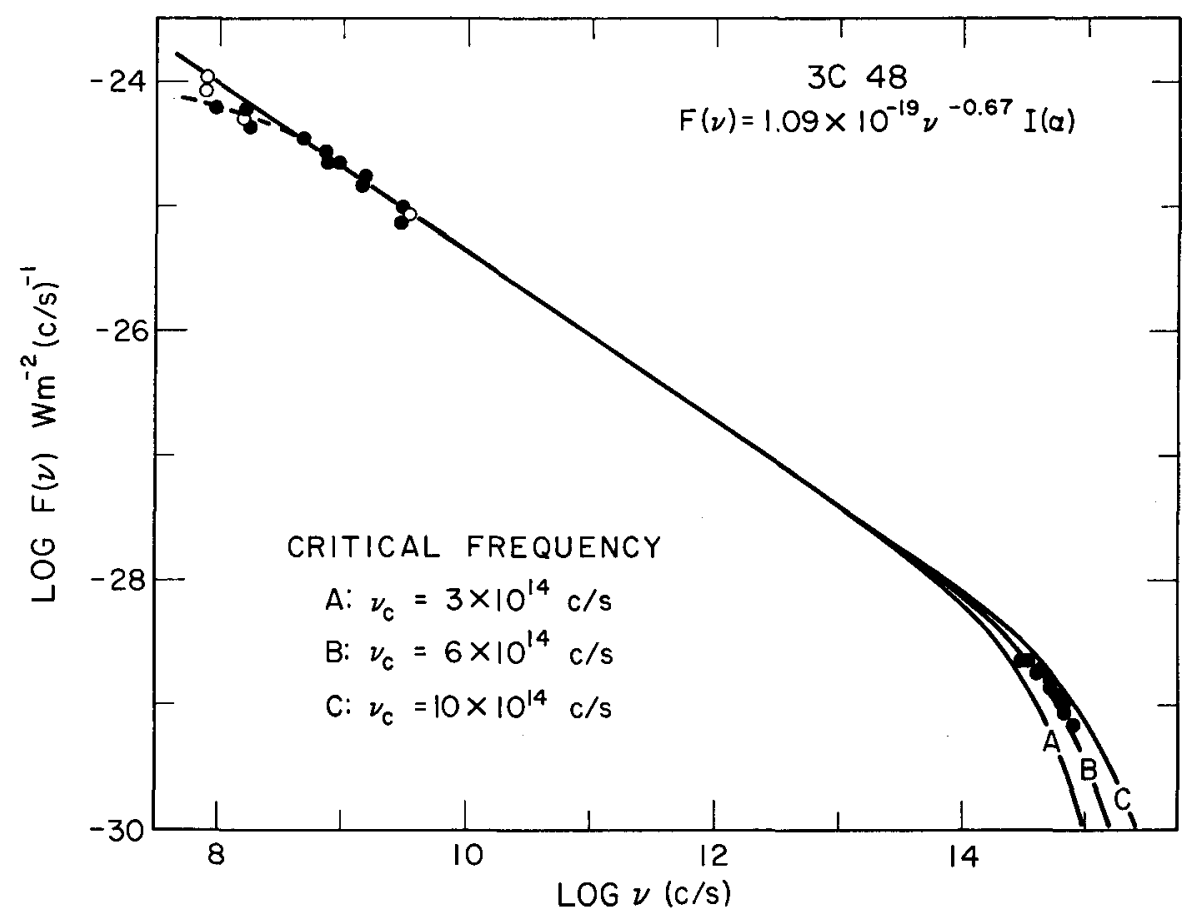

FIG. 9. The energy spectrum, per unit frequency interval, of $3 C 48$ from the radio to the optical region. Three theoretical synchrotron curves are shown for various choices of the critical frequency at the high-energy end.

connected in some QSS concerns the radio source MSH 14-121 (Véron, 1965), which is a radio double separated by $20^{\prime \prime}$, and where a central blue optical object exists which is the optical quasar. The clear physical separation between the regions of optical and radio emission proves that the radio and optical emission must be decoupled here and must, therefore, result from different electron distributions. As Professor Ryle points out, many other QSS are radio doubles and are, therefore, similar to MSH 14-121.

However, the strongest clue that the optical radiation may after all be of synchrotron origin comes from data on optical variation and polarization.

\section{OPTICAL VARIATION}

Nearly every adequately observed QSS shows optical variations. The data for 3C 273 and for 3C 48 are so well known that they need not be here reviewed (Smith and Hoffleit, 1965; Matthews and Sandage, 1963).

The largest outburst so far observed occurred in $3 \mathrm{C} 446$, where an increase in intensity by a factor of 20 was found in June 1966. The broad-band UBV colors changed between pre- and post-outbursts, and the two emission lines of CIV (1550) and CIII 


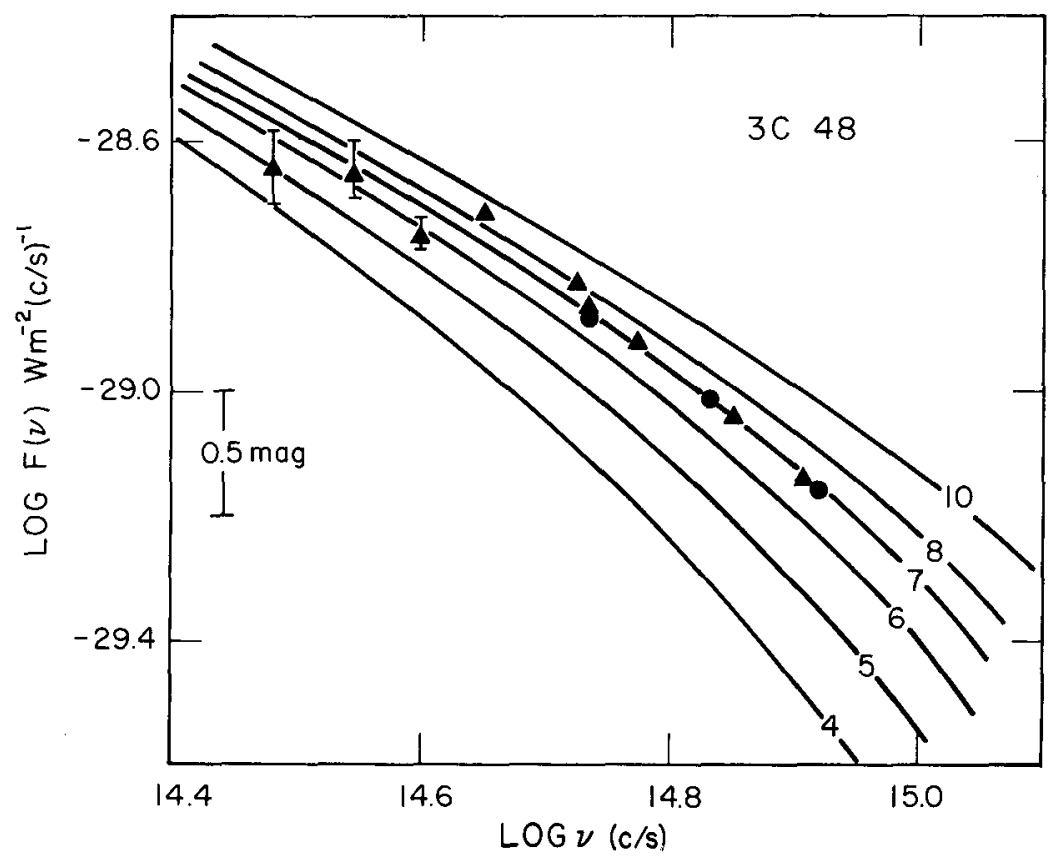

FIG. 10. Detail of Figure 9 in the optical region, with curves of critical frequency marked. Discussion is from Astrophys. J., 138, 1963, 30. The closed circles are from UBV data. The triangles are from measurements of W.A. Baum.

(1909), which were bright and easily visible on spectrograms by Schmidt before outburst, all but disappeared at maximum light. Westphal, Strittmatter, and Sandage showed that the absolute intensity of the lines remained constant and were swamped by the increased continuum during outburst - results that were confirmed and improved by Oke and Wampler. The fact that the lines remained constant while the continuum changed is the first direct proof of a layered quasar model where the two types of radiation are emitted in different regions of the object.

Kinman et al. (1966) followed the outbursts in 3C 446 and found the most remarkable light curve shown in Figure 11. The rate of dimming, in the rest frame of the quasar, was about $0.2 \mathrm{mag} /$ day, with a total drop of 1.6 magnitudes in 8 days. Even greater rates were observed in $3 C 345$ by Goldsmith and Kinman (1965). Other active QSS include 3C 2, 3C 43, 3C 196, 3C 279, and 3C 454.3.

The connection of these results with the question of optical synchrotron emission can be made using the recent observations of Kinman and of Visvanathan that a number of active sources are highly polarized. $3 \mathrm{C} 446$ has a polarization of about $30 \%, 3 \mathrm{C} 279$ of $15 \%, 3 \mathrm{C} 345$ of $8 \%$, and $3 \mathrm{C} 454.3$ is very high but the final value is not yet known. Kinman has presented preliminary evidence that the plane of polarization varies as the optical flux changes and, further, that the percentage polarization be- 


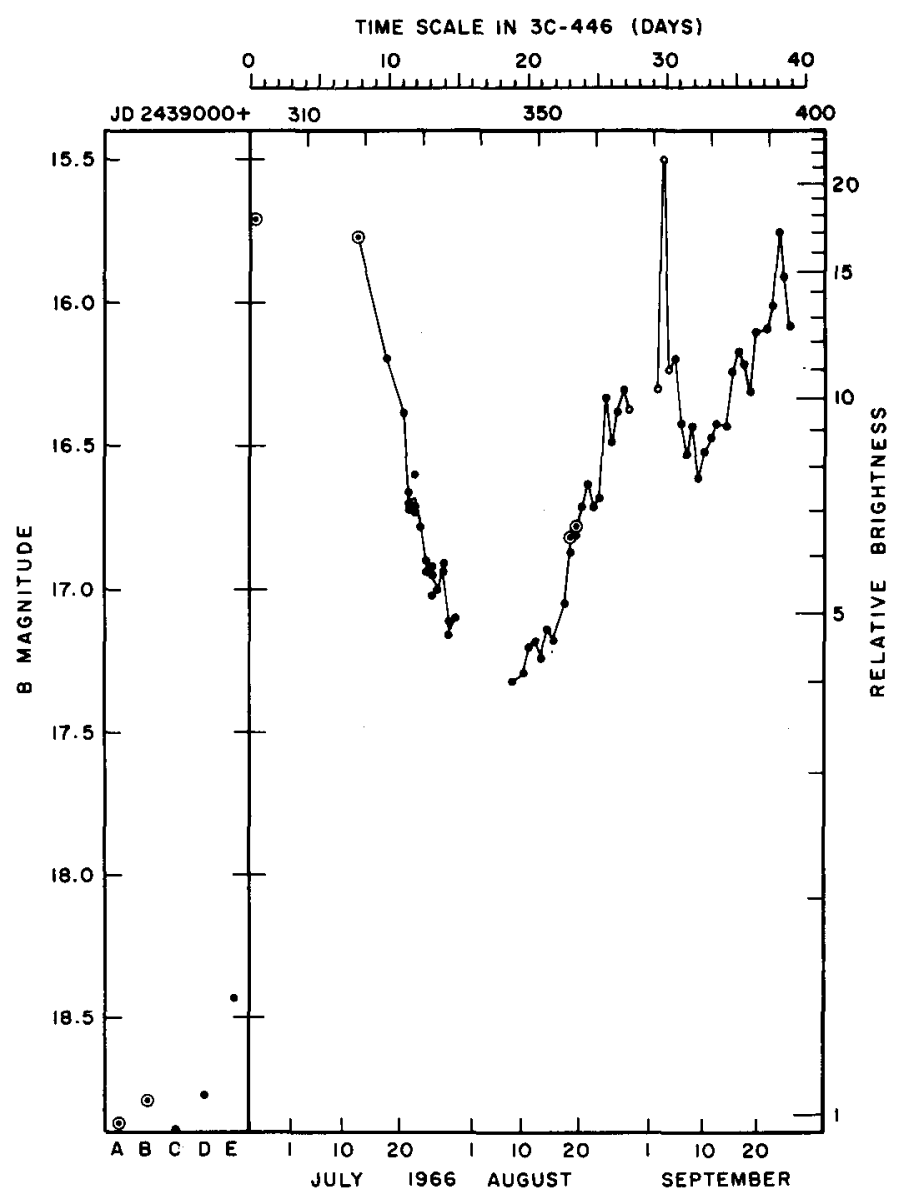

FIG. 11. The light curve for $3 C 446$ according to Kinman et al. (1966). Dots are photographic determinations; circled dots are photoelectric measurements.

comes small when the QSS becomes quiescent. The data are quite suggestive that (1) at least the variable part of the optical radiation may be due to synchrotron emission, (2) that the magnetic fields are not randomly tangled on the scale of the region emitting the variable radiation, and (3) that the aspect of the field seen either by the electrons or by the observer changes with time. The suggestion is that the outburst may be caused by beamed photons due to bursts of fresh electrons traveling along semi-regular field lines. As the aspect of the field changes, due either to rotation of the object or to different spatial regions of curved field lines that are sampled by the electron bursts as the particles travel outward, the intensity and the polarization of the radiation must change. During the quiescent phase, the net polarization may be low because of an integration over the entire body. In this regard, Kellerman's report (Prague IAU, 
1967) is important in that the polarization of the radio flux from quasars is greatest during those times when the radio flux is changing most rapidly, which is analogous to the optical case.

\section{OPTICAL VARIATIONS AND THE DISTANCE}

In the past, the rapidity of the light variations has raised doubts about the cosmological origin of the red-shifts. Terrell has shown that the time-scale of the optical fluctuation of the order of days requires that the linear size of that part of a QSS which is outbursting must be less than a few light days across. A few years ago this was considered the death knell of cosmological red-shifts because of (a) the seemingly impossibly small angular size which was required, and (b) the apparent impossibility of maintaining synchrotron radiation for any appreciable time in such a compact region of high energy density due to the inverse Compton losses of the electrons as they collide with their own photons. This last effect was pointed out by Hoyle et al. (1966).

Both views are perhaps pessimistic. Radio angular-diameter measurements from the long base-line interferometer experiments of the Jodrell Bank group and the Royal Radar Establishment in England, and by the Green Bank workers in the U.S.A., together with the radio-scintillation results at Cambridge and at Arecibo, Puerto Rico, show that all active QSS have angular diameters less than $\simeq 0.02^{\prime \prime}$. These measurements are upper limits and, therefore, do not yet constitute an argument against the cosmological distances. Furthermore, it has now been shown that the Seyfert-like nuclei of N-type galaxies exhibit optical variations like those of QSS (Oke, 1967; Sandage, 1967), and these systems are known to be at the Hubble distance. This observation is perhaps the most powerful argument now available against the necessity for rapid optical variations to demand the objects be local.

The argument of energy loss by inverse Compton effect appears to be overcome by a proper arrangement of the magnetic fields and electron trajectories (Woltjer, 1966). Although the requirements for such a regular field may seem severe, a field of this type is, in fact, apparently demanded by the high degree of optical polarization observed by Kinman and Visvanathan.

\section{The Number of Quasars: Radio-Quiet QSS}

The bulk of the quasi-stellar objects are fainter than the flux limit of present radio surveys, and must be found by optical methods. The isolation of 'radio-quiet' quasars came from the first attempts in 1964 to identify radio quasars by the powerful two- or three-color photographic method invented by Haro, making use of the ultraviolet excess relative to normal stars which these objects possess. During a special photographic survey for radio quasars, many blue stellar objects situated far from the radio positions were found, spread over plates taken with the Mount Wilson 100-inch and 
the Palomar-Schmidt. After some time, it was realized that these objects were similar to those found in earlier Schmidt surveys by Haro and Luyten in 1961 in the magnitude range $15<B<19$.

Subsequent spectrograms proved that a number of the blue objects had intense, broad, red-shifted emission lines, together with the other attributes of normal QSS, save measurable radio flux as bright as 9 flux units. The color distribution of these radio-quiet red-shifted objects is the same as the QSS in the U-B, B-V plate (Figure 6), and quite different from the compact $\mathrm{N}$-type galaxies. The objects look completely stellar on the largest available plate-scale, many vary in optical brightness, they have no measurable proper motion, and the absolute luminosities for the available sample are indistinguishable from those of radio QSS.

Figure 12 is a reproduction of parts of Palomar-Schmidt plates showing the radio QSS $3 C 9\left(\Delta \lambda / \lambda_{0}=2 \cdot 012\right)$, and the first radio-quiet quasar BSO1 $\left(\Delta \lambda / \lambda_{0}=1 \cdot 24\right)$. The two objects are similar in every optical property.

Not all faint $\left(B>16^{m}\right)$ blue stellar objects found in high galactic latitudes are quasars. Spectra by Kinman, Greenstein, and Sandage show that nearby white dwarfs are the most frequent contaminant in any faint blue-star sample. This was predicted

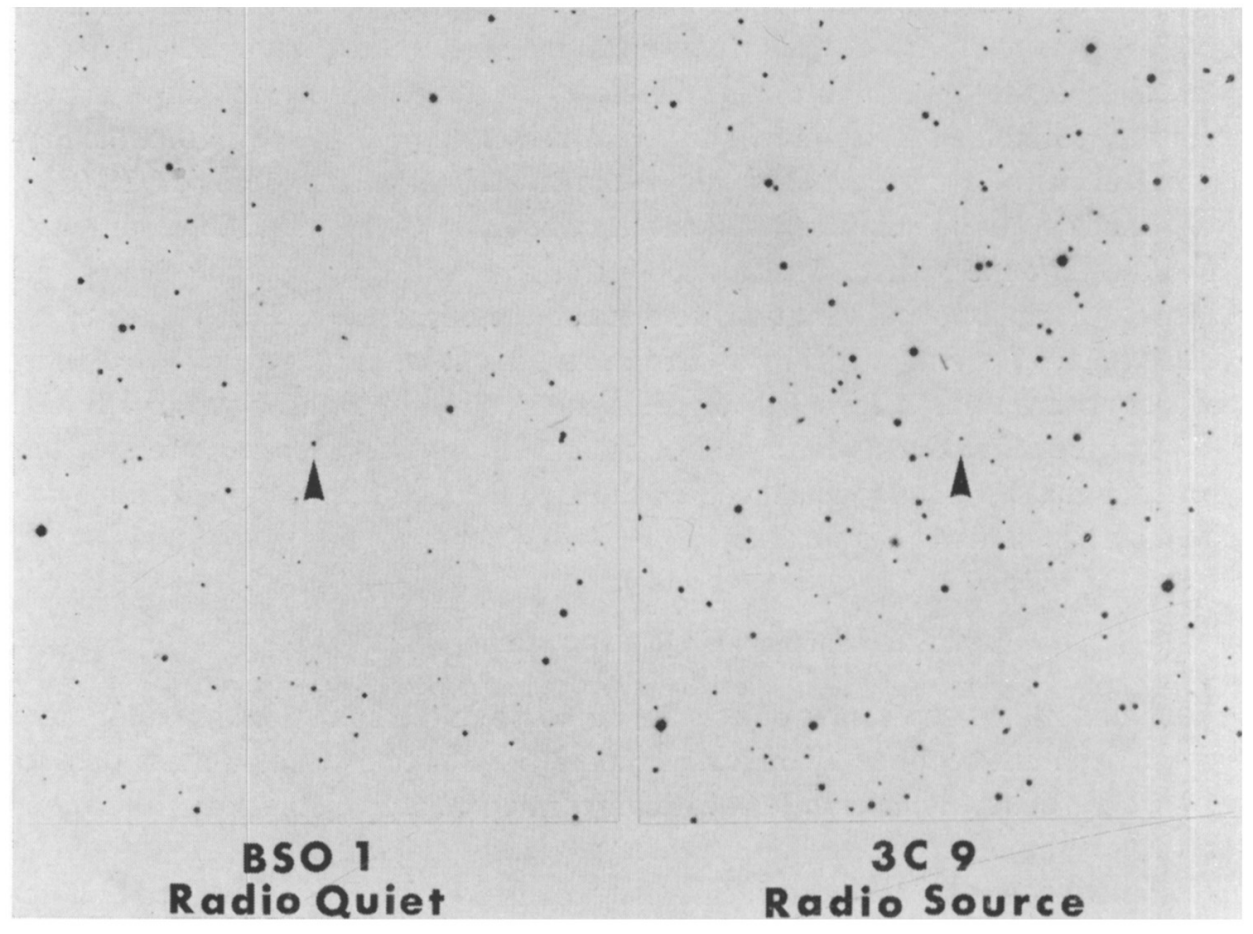

FIG. 12. Comparison of photographs of the radio quasar $3 C 9$ and the radio-quiet quasar BSO1 made with the Palomar 48-inch Schmidt telescope. 
by Kinman (1965) in a discussion which showed that my earlier expectation of most of the Haro-Luyten objects as quasars was an overestimate by about a factor of 4 . Luyten has experimentally separated some of the white dwarfs from potential QSO's by proper-motion studies. The method was particularly successful in a field at $1^{\mathrm{h}} 36^{\mathrm{m}}$, $+6^{\circ}$, where we found (Sandage and Luyten, 1967) about one white dwarf per square degree to $B \simeq 18.1 \mathrm{mag}$., and a minimum number of 0.3 radio-quiet quasars per square degree to the same limit. Figure 13 shows the spectra of 3 of the 11 radio-quiet quasars found in this field.

We have estimated, on the basis of photometry of blue objects in a field studied by $\mathrm{N}$. Richter near Messier 3, that between 1 and 3 QSO exist per square degree to $B \simeq 19 \cdot 7^{m}$. This gives a total of perhaps $10^{5}$ such objects over the plane of the sky to a relatively bright optical limit. Similar studies by Véron and by Van den Bergh show that the number of potential blue objects to $B \simeq 20$ is greater than $10 / \square^{\circ}$, among which must be white dwarfs and QSO. It thus appears likely that the number of genuine quasars to $B=20^{m}$ is very large indeed, as has already been shown to $B \simeq 18^{m}$ from available spectrographic results by Lynds, Kinman, Hiltner and his co-workers, and myself. The existence of the objects is now established, and their large number is germane to the current debate over distances of the QSS.

'Radio-quiet' means only that the object is a weak enough emitter to remain undetected with the largest available radio telescopes. Assessment of the selection effects and of the sensitivity of present equipment shows that many 'radio-quiet' quasars can, in fact, be radio emitters at the faint end of the radio-luminosity function, such that

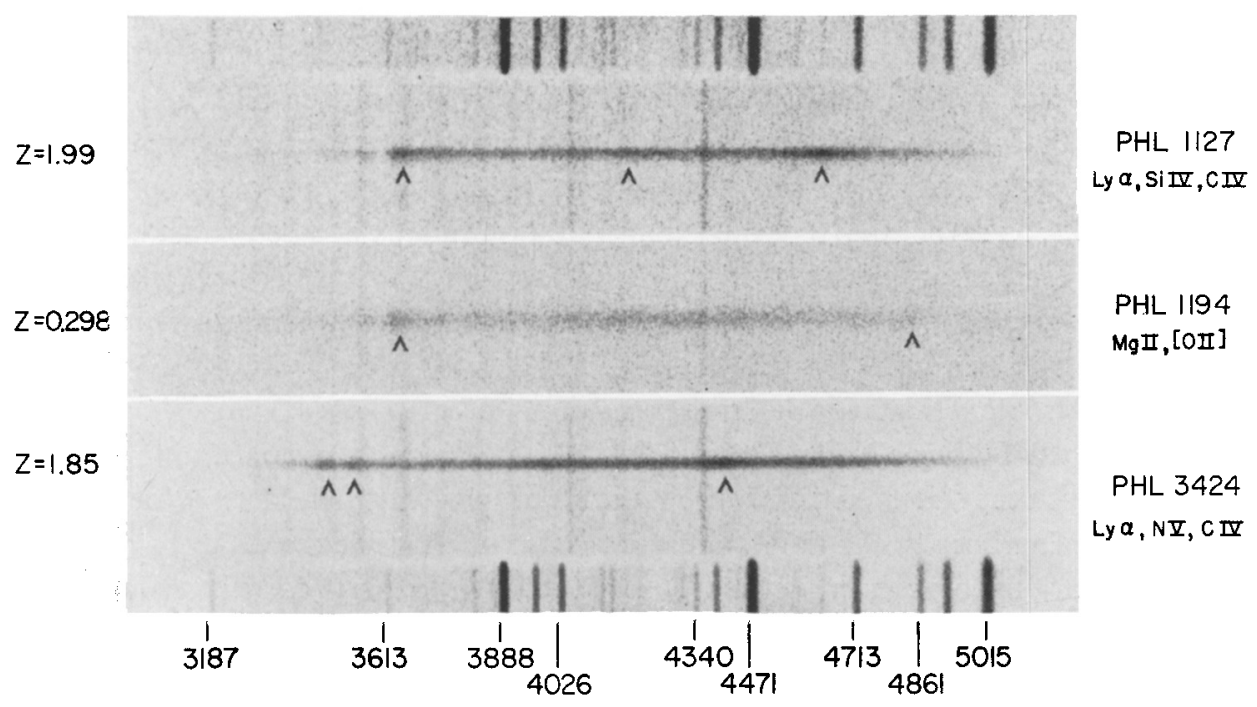

FIG. 13. Spectra of three radio-quiet quasars in the Haro-Luyten catalogue located in a systematic study of the area marked in Figure 5 at $1^{h} 36^{m},+6^{\circ}$. Reproduced from Astrophys. J., 148, $1967,767$. 
$L_{\mathrm{R}} \leqslant 10^{43} \mathrm{ergs} / \mathrm{sec}$ (absolute power between $10^{7}$ and $10^{10} \mathrm{~Hz}$; see Sandage and Luyten, 1967, Figure 4). However, if $10^{5}$ quasars do, in fact, exist over the entire sky, most will be fainter than $2 \mathrm{f}$.u. at $178 \mathrm{Mc} / \mathrm{sec}$, which is the limit of the $4 \mathrm{C}$ catalogue. This is because the $4 \mathrm{C}$ contains $10^{4}$ sources, $30 \%$ of which may be QSS, giving only $3 \times 10^{3}$ objects - far short of the $10^{5}$ estimated number.

\section{Interpretation of the Red-Shift}

Faced with the heretofore unknown combination of enormous total power and small linear size which result if QSS are at the Hubble distance, Terrell (1966, summary) proposed as an alternative to the cosmological explanation of the red-shifts that quasars are nearby objects that have been ejected from the center of our Galaxy in a violent event. He interpreted the red-shifts as Doppler motion with velocities reaching $0 \cdot 8 c\left(\Delta \lambda / \lambda_{0}=2\right)$, assumed to originate in the initial acceleration processes associated with the explosion. Another version of a local hypothesis is available from Hoyle and Burbidge (1966) where ejection of QSS from a nearby radio galaxy such as M87 or NGC 5128 is contemplated.

Although each of these suggestions does have the property of reducing the total power of any given quasar by the square of the ratio of the Hubble distance to the 'local' distance, the advantage is empty because radio galaxies such as Cyg A and $3 \mathrm{C}$ 295 (which are at the Hubble distance) already radiate as much power in radio wavelengths as do the most powerful quasars when they are put at the Hubble distance. Thus the energy problem is not solved because it is clear that radio galaxies themselves present the same dilemma of enormous radio power. Furthermore, each local hypothesis itself runs into energetic difficulties when the problem is seen in toto, if there are as many quasars as the optical studies of radio-quiet QSS suggest. Setti and Woltjer have shown that a very energetic event is required to accelerate so many objects to their observed velocities. The argument is that, since a minimum mass per QSS is about $10^{5} M_{\odot}$, as earlier derived, the explosion in our galactic system must have had at least $10^{61} \mathrm{ergs}$, assuming $10^{5}$ QSS and $100 \%$ efficiency of energy conversion into motion. This is considerably more than the energy of the brightest quasar on the cosmological hypothesis.

The same problem exists for the hypothesis that QSS are ejected from a single radio galaxy, such as M 87 or NGC 5128, in addition to other problems concerning the lack of anisotropy in the observed distribution, the lack of observed blueshifts (Noerdlinger et al., 1966; Faulkner et al., 1966), and the resulting long time-scale.

Although arguments concerning 'reasonable' limits to the energy carry little weight because the entire QSS phenomenon is itself still so manifestly strange and no rules of reasonableness are yet available, it is clear from the preceding that the proponents of local hypotheses cannot use the energy argument to support their position because they themselves are on the high side of the energy scale if all QSS come from a single parent body. 
However, far more serious than the energy totals is the problem, already discussed, of the observed number of quasars at a given redshift, $z$, in range $\Delta z$. The characteristics of all known natural explosions is that the distribution of velocities among the given fragments is such that, some time after the explosion, most of the pieces will be in the low-velocity end, and hence close to the seat of the explosion. Only a few of the fragments will be given excessively large speeds. These, of course, will be found farthest from the explosion centre as time progresses. In no case will the fragment distribution approach that of a uniform density such that successive shells of radii $r_{i}$, each of volume $4 \pi r_{i}^{2} \Delta r_{i}$, will each have the same number of fragments. Even less likely is the case of a positive density gradient outward. And yet such is the observed situation with quasars, according to the recent work of Kafka and of M. Schmidt.

Only by making the most ad hoc assumptions can these observed facts be understood on a local hypothesis, whereas the explanation is natural and convincing with normal cosmological models and a reasonable variation of the frequency of production of QSS with cosmic time.

Rather than recite further difficulties of the local hypotheses, a more positive approach is to describe new observations germane to the opposite conclusion. There now exist new data which seem to support the cosmological interpretation of the redshifts. The tests all rest, ultimately, on the fact that radio galaxies are, themselves, at the Hubble distance, as will now be shown.

\section{Evidence for the Cosmological Distances of Radio Galaxies and Quasars}

Figure 14 shows the most recent data on the red-shift-apparent magnitude relation for the brightest galaxy in each of 41 clusters of galaxies. In 29 of the clusters, the brightest galaxy is not a radio source, while the opposite is true in the remaining 12 clusters, which include the famous cases of 3 C 295, Per A (NGC 1275), and 3C 40 (NGC 545).

Plotted in Figure 14 as abscissa is the photoelectric $V$ magnitude corrected for (1) aperture effect to an isophote of about $25^{m}$ per square second of arc, (2) $K$ dimming due to red-shifting the energy distribution through the fixed measuring band, and (3) galactic absorption, $A_{V}$, assuming a cosecant law with a galactic half-thickness of $A_{V}\left(\frac{1}{2}\right)=0 \cdot 18^{m}$. Red-shifts, corrected for galactic rotation, are plotted as ordinate.

The plotted line has not been put through the data as regards slope. It is given a slope of 5 , which is the theoretical value for all cosmological models as $\Delta \lambda / \lambda_{0} \rightarrow 0$, and is the exact value for all $\Delta \lambda / \lambda_{0}$ values in a Friedmann model with $q_{0}=+1, \Lambda_{0}=0$, and the evolutionary correction $\mathrm{d} L / \mathrm{d} t=0$. The data follow this line with remarkable fidelity. The dispersion $\Delta M$ is exceedingly small $\left(\sigma_{M}=0 \cdot 28^{m}\right)$, which shows that the brightest galaxy in a cluster defines a standard candle to within $\pm 30 \%$ in absolute luminosity - a truly remarkable fact.

The important point to recall about Figure 14 is that the line represents the linear 


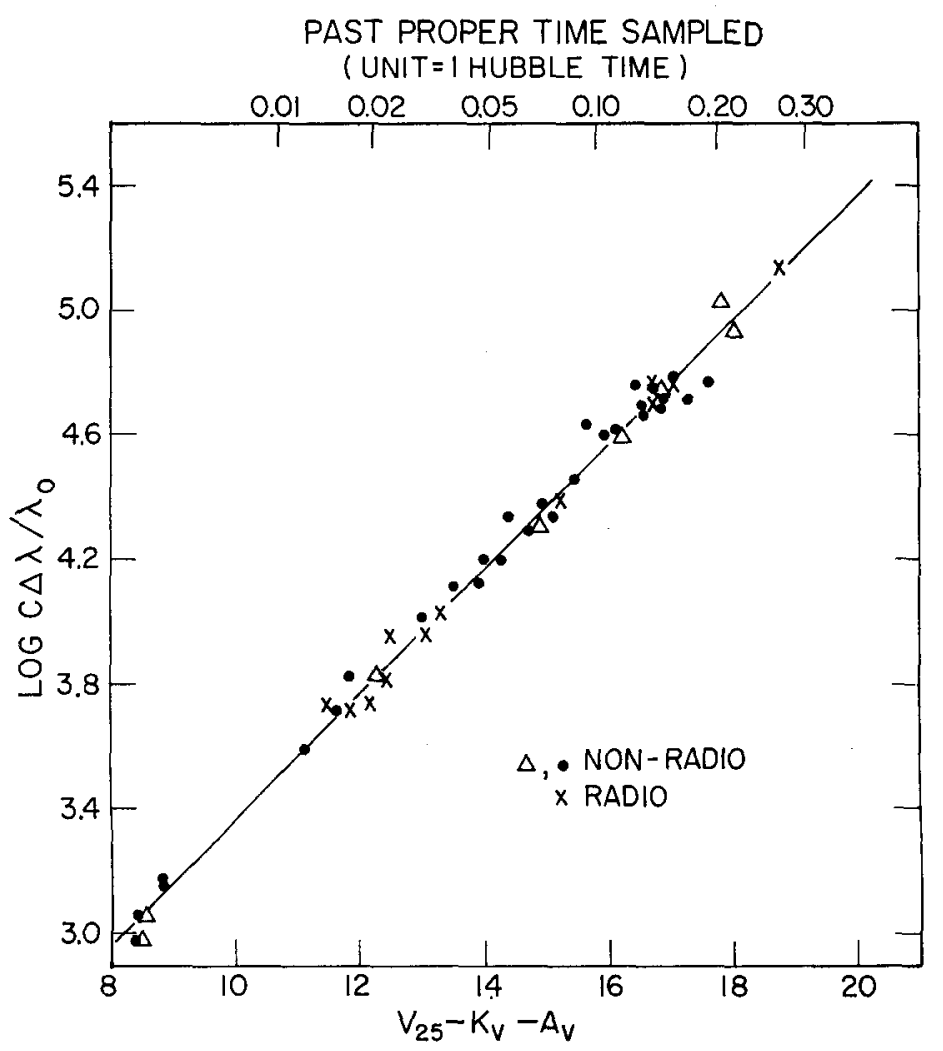

FIG. 14. The Hubble diagram of first-ranked cluster galaxies in 41 clusters. Dots are non-radio galaxies, crosses are radio galaxies, and open triangles are data by Baum for eight clusters, some of which are duplicated. All photometry is photo-electric and was obtained with the 200-inch Hale telescope. Correction for aperture effect, for $K$ dimming, and for galactic absorption have been applied. The line has a slope of 5.0 and has been fitted to the data only in zero point.

Hubble expansion law. The fact that all the galaxies obey the line proves beyond doubt that they are at their Hubble distances. The same conclusion follows for radio galaxies in general, as Figure 15 demonstrates. Here data for 65 radio galaxies are plotted. While only $30 \%$ of the galaxies are members of clusters, the conclusion is the same as for Figure 14. Again the line has the theoretical slope of 5, and it is evident that the galaxies obey linear red-shift relation. The increased scatter in Figure 15 compared with that of Figure 14 is undoubtedly due to a less homogeneous statistical sample, and therefore to an increased spread in absolute magnitude $M$. We are here sampling deeper into the optical luminosity function of radio galaxies for objects shown in Figure 15. Yet this diagram is still remarkably tight, proving that a necessary condition for a galaxy to be a strong radio source is that it be in the class of the most luminous galaxies known, namely, the several brightest members of clusters. 


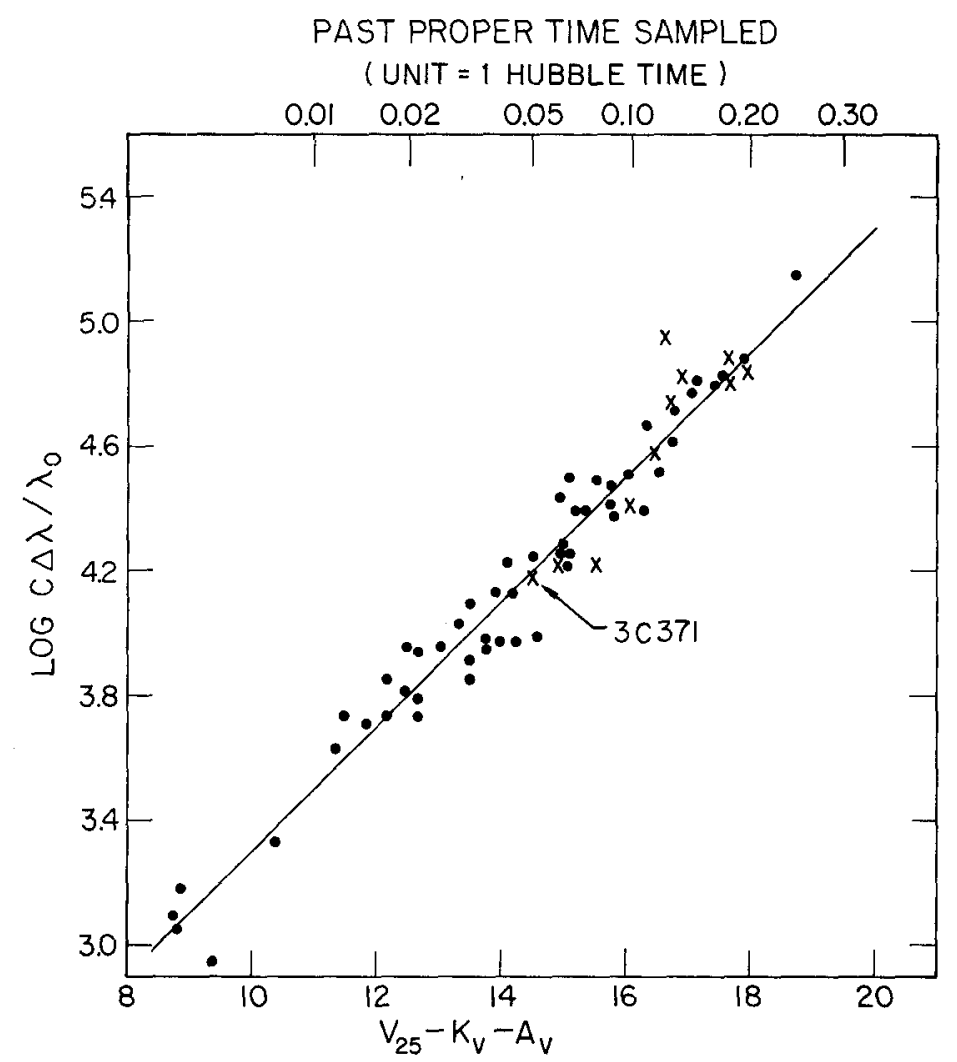

FIG. 15. Same as Figure 15 for all identified radio galaxies with red-shifts and photoelectric data known to August 1967. The dots are normal elliptical galaxies, crosses are $N$-systems. There is no evidence that the compact $\mathrm{N}$-galaxies deviate from the Hubble law.

It should here be emphasized that all of the galaxies shown in Figures 14 and 15 are elliptical systems. No spirals are amongst them. In Figure 14 this is because, as known since Hubble's work in 1936, most galaxies in the great clusters are ellipticals, almost without exception. The same is true in Figure 15, because the fact that emerged from the optical identification work was that the most powerful radio galaxies are, almost without exception, pure population-II systems.

Figures 14 and 15, taken together, show that all radio galaxies studied so far obey the Hubble law, and are therefore at their cosmological distance. The same conclusion follows from study of angular diameters, where the data for radio galaxies and firstranked cluster members obey $\theta \propto z^{-1}$.

With radio galaxies now established to be at their Hubble distances, we can proceed to three crucial tests concerning quasars. The first is due to Heeschen (1966) and is shown in Figure 16, where the radio surface brightness, $B$, of those radio galaxies and QSS with resolved radio-disks is plotted against the absolute radio power, $L_{\mathrm{R}}$, 


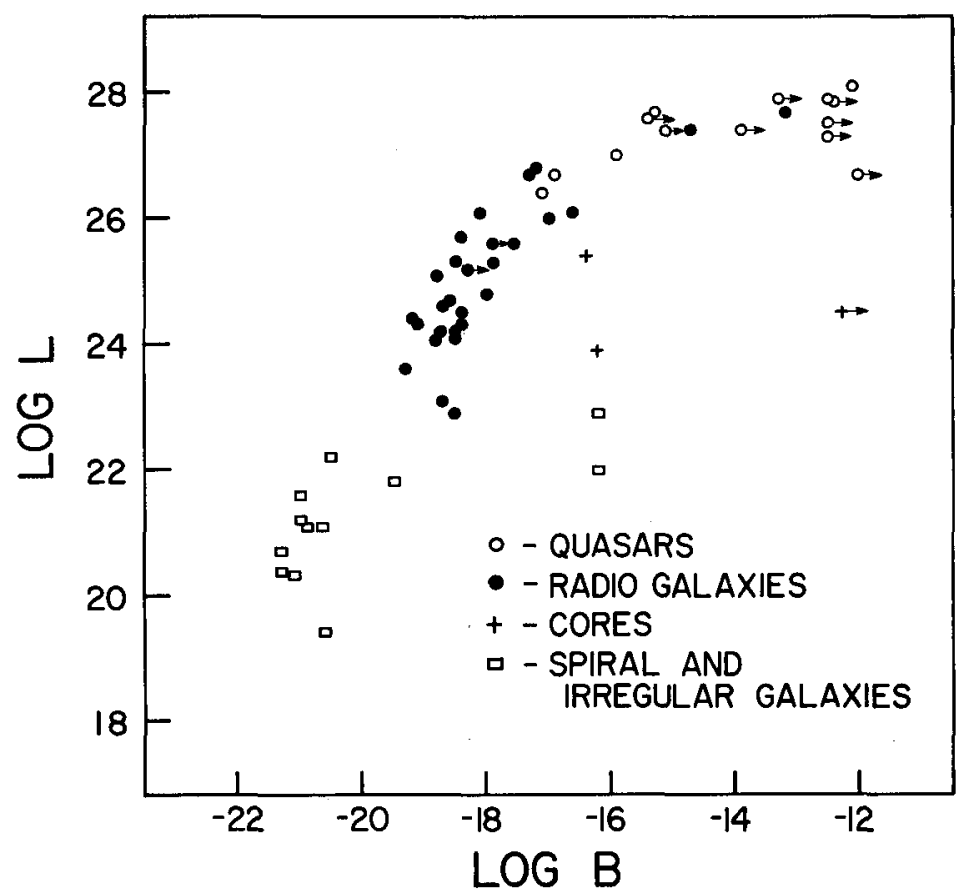

FIG. 16. Heeschen's diagram of the correlation of surface brightness and absolute radio power for quasars and radio galaxies, calculated as if all objects are at their Hubble distances. Continuity between $Q S S$ and radio galaxies would be destroyed if the red-shifts of QSS are not cosmological.

calculated as if all sources are at their Hubble distances. Because $B$ is independent of distance and $L_{\mathrm{R}}$ depends on the distance squared, the observed correlation and continuity would be destroyed if the QSS did not follow the Hubble law. Heeschen's result appears to be a crucial experiment which shows that quasars do indeed obey the redshift-distance relation of the expanding universe.

A second set of data on the linear-size distribution of radio splitting for galaxies and QSS has already been mentioned by Professor Ryle. The distances between the radio components of galaxies are distributed between 1 and $450 \mathrm{kpc}$. The same range is covered by the double radio components of QSS if they are at the Hubble distance, but not if the QSS are local (Kellerman, 1966; Hazard 1967). These and newer data are particularly well shown in the diagrams discussed by Ryle in the first of these lectures.

A third indication of the similarity of quasars and the violent events of galaxies has become known only in recent weeks. The N-type galaxy, 3C 371, shown as a cross in Figure 15 at the smallest red-shift for the $\mathbf{N}$ systems, was discovered to vary in optical luminosity (Oke, 1967; Sandage, 1967) by one magnitude on a long time-scale, and by 15 to $20 \%$ from night to night. Subsequently, the N-type galaxies, 3C $390 \cdot 3$ 


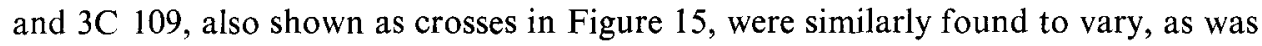
the Zwicky blue compact galaxy I $\mathrm{Z} 1727+50$, which is of the class of radio-quiet analogs of the $\mathrm{N}$-type radio galaxies. All these $\mathrm{N}$ galaxies obviously follow the Hubble law (Figure 15), and yet they vary in a similar fashion to QSS. They form the single counter-examples needed to prove that rapid variation of optical flux does not require that the objects need be local.

The continuity of properties between the Seyfert nuclei, the N-type galaxies, and the quasars now seems complete. They all appear to be manifestations, in different degrees, of the same type of non-thermal violent event taking place in the nuclei of galaxies. The physical cause still eludes us. The reason why only the most massive galaxies known can become strong radio sources is equally unknown. What is clear phenomenologically is that the violence of the event itself possesses a great dispersion in optical-power level, as shown in the next diagram.

Figure 17 is the Hubble diagram for radio galaxies and for QSS. The large spread in the distribution of the triangles is evident. The observed dispersion in absolute luminosity of QSS is at least 4 magnitudes. It is significant that the QSS merge into the radio galaxies in $M_{V}$. The fact that no QSS is known to exist to the right of the distribution of galaxies may be significant. Perhaps we are justified in suggesting that when the violent event giving rise to the ultraviolet, non-thermal emission is relatively mild, it cannot outshine the stellar component of an underlying galaxy, and the system would be classed as $\mathbf{N}$. If the outburst is still weaker, it can only dominate the nuclear regions, and we observe a Seyfert galaxy.

Finally, it should be pointed out that the lack of a strong correlation among the triangles in Figure 17 of the red-shift and apparent magnitude surely does not indicate a failure of QSS to follow the linear Hubble law. Such a conclusion is incorrect when the sample of objects considered has a large dispersion in intrinsic luminosity. The steepening of the upper envelope of the QSS distribution with increasing red-shift is, in fact, what is expected for a sample of objects with no upper cut-off to the luminosity function as the volume of space sampled becomes larger (higher $z$ values). This is the Scott effect (Scott, 1957). It is not justified, as some have done, to conclude that the spread of the triangles in Figure 17 implies that the QSS show no red-shiftdistance effect. When the selection effects in the presence of a large intrinsic dispersion are considered, the opposite would appear to be the case.

\section{Epilogue}

Four years ago high hopes were expressed that quasars would play a dominating role in the solution of the geometrical cosmological problem. Although the red-shifts reach enormous values of $\Delta \lambda / \lambda_{0} \simeq 2$, which means we look back $80 \%$ of the way in time to a singularity of the Friedmann universe, we cannot separate out amongst the intrinsic spread of absolute optical power the effects of deceleration which, through 


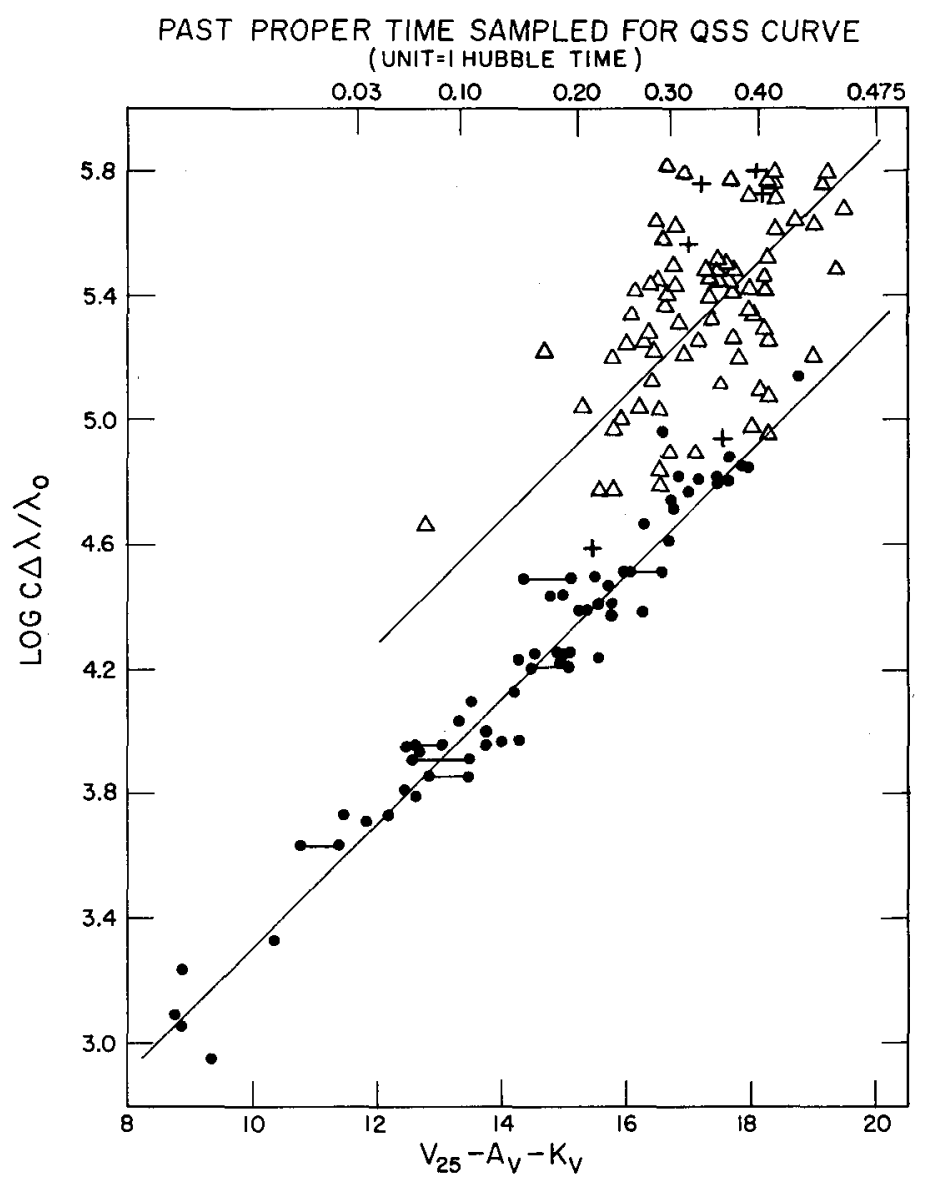

FIG. 17. The Hubble diagram for radio galaxies (dots), radio quasars (triangles), and radio-quiet quasars (crosses). Horizontal bars connect points for dumbbell galaxies which represent the combined light of both components and the light of the brightest component. The time into the past to which we look at a given red-shift is shown along the top in units of one Hubble time, which is $\mathrm{H}_{0}^{-1} \simeq 13 \times 10^{9}$ years with the present calibration.

Einstein's coupling of motion and geometry, leads to a value of the spatial curvature of the world. Disappointing as this may be, quasars and radio galaxies have nonetheless provided something quite unique in the physics of astronomical structures. Instead of serving only as markers of an underlying geometry, which quasars would have done had their intrinsic luminosities shown less spread, they now provide an ontogenetic mystery whose solution may lead us even deeper into historical events of the distant past. If Schmidt's belief is true, that creation of quasars is strongly timedependent, being more prolific near the birthday of the world, then a new dimension to cosmogony is opened up. Radio galaxies, because of their remarkable statistical stability of absolute luminosity, still appear capable to serve as the test objects - the 
standard candles - the fundamental probes for geometrical cosmology. High hopes, therefore, still remain that the deceleration parameter will be determined from data such as those of Figures 14 and 15. But the even more fundamental problems of the explosion physics remain.

Nowhere in this lecture has the name of Ambartsumian appeared. He foreshadowed much of what has here been said. Ten years ago he began to emphasize the role of nuclei of galaxies. Consistently, at the Solvay conferences, at the Berkeley IAU and at numerous symposia, he, almost alone at first, suggested that violent processes were indeed occurring in galactic nuclei, and that astronomers should take full heed. The fulfillment of his prophecy is only now beginning. No astronomer would today deny that mystery does indeed surround the nuclei of galaxies, and the first man to so realize the rich reward in store was Victor Ambartsumian.

The events preceding the violent release of energy in QSS, $\mathrm{N}$ galaxies, and Seyfert nuclei, and the reasons for the instabilities still remain a cryptic mystery. But, at least we know now somewhat better the questions to be solved - questions which drive the theoreticians to think, and the observers to the dark and quiet of their telescopes, both radio and optical, to help unravel the greatest problem known to man - the scientific story of creation revealed through the history of galactic systems and their predecessors.

\section{References}

Burbidge, E. M., Burbidge, G.R. (1965) Astrophys. J., 142, 1351.

Burbidge, E. M., Burbidge, G.R., Rubin, V.C. (1964) Astrophys. J., 140, 942.

Epstein, E.E. (1965) Astrophys. J., 142, 1285.

Faulkner, J., Gunn, J.E., Peterson, B.A. (1966) Nature, 211, 502.

Goldsmith, D.W. Kinman, T.D. (1965) Astrophys. J., 142, 1693.

Greenstein, J. L., Schmidt, M. (1964) Astrophys. J., 140, 1.

Hazard, C. (1967) New York Third Texas Meeting on Cosmology.

Heeschen, D.S. (1966) Astrophys. J., 146, 517.

Hoyle, F., Burbidge, G. R. (1966) Astrophys. J., 144, 534.

Hoyle, F., Burbidge, G. R., Sargent, W.L.W. (1966) Nature, 209, 751.

Kellerman, K.I. (1966) Astrophys. J., 146, 621.

Kinman, T.D. (1965) Astrophys. J., 142, 1241.

Kinman, T.D., Lamla, E., Wirtanen, C.A. (1966) Astrophys. J., 146, 964.

Low, F.J., Johnson, H.L. (1965) Astrophys. J., 141, 336.

Lynds, C.R. (1967) Astrophys. J., 147, 396.

Lynds, C.R., Sandage, A. (1963) Astrophys. J., 137, 1005.

Matthews, T. A., Sandage, A. (1963) Astrophys. J., 138, 30.

Matthews, T. A., Morgan, W.W., Schmidt, M. (1966) Astrophys. J., 140, 35.

Miller, W.C. (1955) Astr. Soc. Pacific Leaflet No. 314.

Noerdlinger, P.D., Jokipii, J.R., Woltjer, L. (1966) Astrophys. J., 146, 523.

Oke, J. B., (1967) Astrophys. J., 150, L5.

Sandage, A. (1967) Astrophys. J., 150, L9.

Sandage, A., Luyten, W.J. (1967) Astrophys. J., 148, 767.

Sandage, A., Miller, W.C. (1964) Science, 144, 405.

Scheuer, P.A.G., Wills, D. (1966) Astrophys. J., 143, 274.

Schmidt, M. (1968) Astrophys. J., 151, 393. 
Scott, E. L. (1957) Astron. J., 62, 248.

Setti, G., Woltjer, L. (1966) Astrophys. J., 144, 838.

Smith, H.J., Hoffleit, E.D. (1965) Gravitational Collapse and Quasi-Stellar Sources, Chapter 16.

Strittmatter, P., Faulkner, J., Walmsley, M. (1966) Nature, 212, 1441.

Terrell, J. (1966) Science, 154, 1281.

Véron, P. (1965) Astrophys. J., 141, 1284.

Woltjer, L. (1966) Astrophys. J., 146, 597. 